Henrique Silveira Rabelo

Menu Choices with Categories

Brasília

2017 

Henrique Silveira Rabelo

\section{Menu Choices with Categories}

Dissertação apresentada ao Curso de Mestrado Acadêmico em Economia, Universidade de Brasília, como requisito parcial para a obtenção do título de Mestre em Economia

Universidade de Brasília - UnB

Faculdade de Economia, Administração e Contabilidade

Departamento de Economia

Programa de Pós-Graduação

Orientador: Leandro Nascimento

Brasília

2017 
Rabelo, Henrique

Menu Choices with Categories. - Brasília, 2017-

40 páginas

Orientador: Leandro Nascimento

Dissertação de Mestrado - Universidade de Brasília - UnB

Faculdade de Economia, Administração e Contabilidade

Departamento de Economia

Programa de Pós-Graduação, 2017.

1. Categorização. 2. Preferência por Flexibilidade. 3. Preferência sobre Menus. 


\section{Agradecimentos}

Agradeço à Deus. À minha família, pelo suporte e amparo em todos os momentos. Aos meus amigos. À minha namorada Natália, por todo o apoio e paciência nos momentos mais difíceis.

Agradeço aos professores da pós-graduação pelo aprendizado. Em especial aos professores Gil Riella, quem desenvolveu a ideia e conteúdo deste trabalho, e Leandro Nascimento.

Agradeço aos colegas da pós-graduação. Pelos momentos de diversão, estudos e companheirismo. Agradeço em especial à turma de Teoria de Decisão, matéria ministrada pelo professor Gil, a qual foi fundamental para o desenvolvimento da ideia dessa dissertação. 



\section{Resumo}

Este trabalho apresenta uma representação de preferências sobre menus na qual a tomadora de decisão categoriza o conjunto de alternativas. Tendo uma preferência estrita sobre as alternativas, ela escolhe o menu hoje sabendo que amanhã irá escolher a melhor alternativa do menu que pertence a uma categoria escolhida aleatoriamente. A incerteza sobre a relevância de cada categoria no ato de escolha leva a uma preferência por flexibilidade. Serão desenvolvidas representações ordinal e aditiva e será mostrado que aditividade impõe restrições adicionais apenas quando as categorias são disjuntas dois-a-dois.

Palavras-chave: Categorização, Preferência por Flexibilidade, Preferência sobre Menus. 



\section{Abstract}

This work presents a representation of preference over menus in which the decision-maker categorizes the set of alternatives. Endowed with a strict preference over the alternatives, she chooses a menu today knowing that tomorrow she will pick the best alternative in the menu that belongs to a randomly drawn category. The uncertainty about the relevance of each category in the act of choice leads to a preference for flexibility. It will be developed ordinal and additive representations and it will be shown that additivity imposes additional restrictions only when the categories are pairwise disjoint.

Keywords: Categorization, Preference for Flexibility, Preferences over Menus. 



\section{Contents}

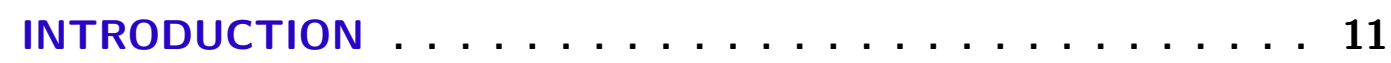

SETUP AND DEFINITIONS $\ldots \ldots \ldots \ldots \ldots$

WEAK CATEGORIZATION $\ldots \ldots \ldots \ldots \ldots$

4

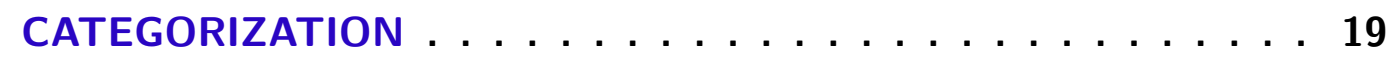

PREFERENCE FOR FLEXIBILITY AND CATEGORIZATION $\ldots 23$

LITERATURE DISCUSSION $\ldots \ldots \ldots \ldots \ldots$

7

CONCLUSION .................. 27

$\begin{array}{ll}\text { APPENDIX } & 29\end{array}$

APPENDIX A-PROOFS ............. 31

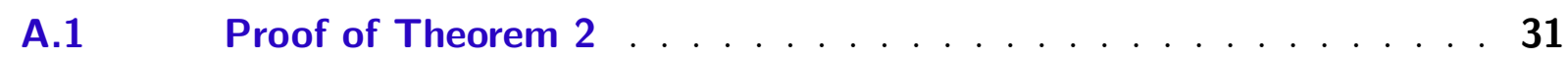

A.2 Proof of Theorem $3 \ldots \ldots 33$

A.3 Proof of Theorem $4 \ldots \ldots \ldots \ldots$

A.4 Proof of Theorem $5 \ldots \ldots \ldots \ldots$

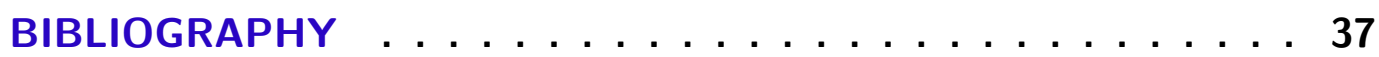





\section{Introduction}

The standard approach to rational choice states that an individual is endowed with a well-defined preference over a set of alternatives and, given a choice problem, chooses the most preferred alternative among all the feasible ones. However, such assumption lacks descriptive appeal and thus many works have attempted to formulate theories of choice that departure from this view, either by weakening the properties of the preference or by weakening the assumption that the individual considers all feasible alternatives for her choice. ${ }^{1}$ Within both approaches, a growing literature accounts for categorization, either by assuming that the individual is able to rank alternatives only within categories (FURTADO; NASCIMENTO; RIELLA, 2017) or assuming that she constrains the choice problem to only some categories (BARBOS, 2010; MANZINI; MARIOTTI, 2012). For its recognized role in decision making (PESKI, 2011; MOHLIN, 2014; AL-NAJJAR; PAI, 2014), categorization has been a prominent subject in decision theory and it is also treated in other related fields, such as finance (BARBERIS; SHLEIFER, 2003), game theory (AZRIELI, 2009; AZRIELI, 2010) and spatial voting (COLLINS et al., 2011; BØLSTAD; DINAS, 2016).

When we assume the individual categorizes the set of alternatives, an issue arises when we deal with dynamic choices. As (KREPS, 1979) points out, "the individual makes decisions which will constrain the choices that are feasible later". In this sense, it would be reasonable to assume that the individual takes into account the fact that she categorize alternatives when facing decisions that affect future choices. If there is uncertainty about which category the individual will want to choose from in the future, then it is natural that she prefer larger sets of alternatives. Within this context, this paper attempts to relate the theory of categorization to the theory of preference for flexibility.

We formulate a theory of rational choice among opportunity sets, or menus, in which the decision-maker (henceforth DM) categorizes the set of alternatives. The analysis is given by a two-stage process of decision making, a choice of a menu and later a choice within the menu. The procedure goes as follows. The DM is endowed with an utility function over the alternatives, but subjectively divide them into categories. The DM is unsure about which category she will want to choose from in the future, so she attributes to each menu a utility value given by the expected value of the maximum utility she can obtain from each category.

The representation developed here decomposes each menu into a vector of indirect utilities, where each component is the maximum utility attained in the menu restricted to 
a specific category. Following (KREPS, 1979), we will work with an ordinal and an additive representation. The ordinal representation simply attributes an utility level to each of those vectors. The additive representation attributes a probability to each category and thus the representation is given in terms of expected indirect utility. Following (FURTADO; NASCIMENTO; RIELLA, 2017), we consider two types of categorizations, one in which categories are arbitrary subsets of the set of alternatives and one in which categories are pairwise disjoint.

The rest of the paper is organized as follows. In the next chapter, we establish the basic framework and present the representations of categorization. In Chapters 3 and 4 we present the main results of the paper and also discuss the two notions of categorization described above. Chapter 5 discusses the link between preference for flexibility and categorization, elucidating the resemblance between our work and the classical work of (KREPS, 1979). In Chapter 6, we discuss some related literature. Chapter 7 concludes the paper. All proofs are presented in Appendix 7. 


\section{Setup and Definitions}

Let $X$ be a finite set of alternatives with arbitrary elements denoted by $x, y, z$, etc.. Given a collection $\mathscr{C}$ of nonempty subsets of $X$, we say that $\mathscr{C}$ is a weak categorization of $X$ whenever $\cup \mathscr{C}=X$. If in addiction all sets $S \in \mathscr{C}$ are pairwise disjoint, so that $\mathscr{C}$ is a partition of $X$, then we say that $\mathscr{C}$ is a categorization of $X$. An arbitrary element $S \in \mathscr{C}$ is called a category. Finally, let $\mathscr{X}$ be the collection of all nonempty subsets of $X$. The elements of $\mathscr{X}$ are called menus and denoted by capital letters $A, B, C$, etc..

Our primitive is a complete preorder $\succsim$ on $\mathscr{X}$. As usual, the asymmetric and symmetric parts of $\succsim$ are denoted by $\succ$ and $\sim$, respectively. We will work with the following notion of ordinal representation:

Definition 1. We say that a relation $\succsim$ on $\mathscr{X}$ has a (weak) categorization representation if there exists an injective function $u: X \rightarrow \mathbb{R}_{++}$, a (weak) categorization $\mathscr{C}$ of $X$ and a strictly increasing aggregator $V: \mathbb{R}^{\mathscr{C}} \rightarrow \mathbb{R}$ such that, for any $A, B \in \mathscr{X}$, we have $A \succsim B$ if, and only if,

$$
V\left(\left(\max _{x \in A \cap S} u(x)\right)_{S \in \mathscr{C}}\right) \geq V\left(\left(\max _{x \in B \cap S} u(x)\right)_{S \in \mathscr{C}}\right)
$$

with the convention that

$$
\max _{x \in \emptyset} u(x)=0 .^{1}
$$

In this case, we say that $\langle u, \mathscr{C}, V\rangle$ is a (weak) categorization representation of $\succsim$. We will also work with a particular case of the representation above, when the aggregator is chosen to be additive in the following sense:

Definition 2. We say that a relation $\succsim$ on $\mathscr{X}$ has an additive (weak) categorization representation if there exists an injective function $u: X \rightarrow \mathbb{R}_{++}$, a (weak) categorization $\mathscr{C}$ of $X$ and a full-support probability measure $\pi$ on $\mathscr{C}$ such that, for any $A, B \in \mathscr{X}$, we have $A \succsim B$ if, and only if,

$$
\sum_{S \in \mathscr{C}} \pi(S) \max _{x \in A \cap S} u(x) \geq \sum_{S \in \mathscr{C}} \pi(S) \max _{x \in B \cap S} u(x)
$$

with the convention that

$$
\max _{x \in \emptyset} u(x)=0 .
$$

$\overline{1}$ By strictly increasing we mean that $\xi \geq \zeta$ and $\xi \neq \zeta$ implies $V(\xi)>V(\zeta)$, for every $\xi, \zeta \in \mathbb{R}^{\mathscr{C}}$. 
In this case, we say that $\langle u, \mathscr{C}, \pi\rangle$ is an additive (weak) categorization representation of $\succsim$. In the representations above, the DM divides the space of alternatives into a collection of categories $\mathscr{C}$. She also has a utility function $u$ that strictly ranks all alternatives in $X$. When choosing a menu today, she acts as if tomorrow she will choose the best element from one randomly chosen category from $\mathscr{C}$. The utility of a given menu is then an aggregation of the utilities of the best elements of each category. The non-additive representations use an arbitrary strictly increasing aggregator, while in the additive representations the aggregator has an expected utility format, given a probability measure over the collection of categories $\mathscr{C}$. We note that the representation assigns a utility of zero whenever there is no element in the menu from the selected category. This can be interpreted as saying that choosing nothing has an strictly smaller ex-ante value for the DM than any other alternative and this value is normalized to 0 .

All the analysis in the paper will rely on the following notion of revealed preference over $X$ :

Definition 3. Given a relation $\succsim$ on $\mathscr{X}$, define the binary relation $\triangleright \subseteq X \times X$ by $x \triangleright y$ if, and only if, $x \neq y$ and either $\{x, y\} \sim\{x\}$ or there exists $A \in \mathscr{X}$ such that $A \cup\{y\} \succ A$ but $A \cup\{x, y\} \sim A \cup\{x\}$.

Intuitively, the relation $\triangleright$ captures the idea that there exists at least one situation in which $y$ loses its value because of the presence of $x$. It turns out that the properties of the relation $\triangleright$ are the distinguishing factor among all the models studied in this paper.

In the next chapter we investigate the conditions that characterize when a relation $\succsim$ on $\mathscr{X}$ admits a weak categorization representation. 


\section{Weak Categorization}

In this chapter, we characterize the relations $\succsim$ on $\mathscr{X}$ that admit a weak categorization representation. We begin with two postulates of (KREPS, 1979).

Axiom 1 (Monotonicity). For every $A, B \in \mathscr{X}$, if $A \supseteq B$, then $A \succsim B$.

Axiom 2 (Submodularity). For every $A, B, C \in \mathscr{X}$, if $A \sim A \cup B$, then $A \cup C \sim A \cup B \cup C$.

(KREPS, 1979) relates the Monotonicity postulate to the notion of preference for flexibility. ${ }^{1}$ The idea is that a decision maker who is uncertain about her future tastes enjoys the flexibility of having more options to choose from in the future. ${ }^{2}$ Submodularity is another postulate that appeared in (KREPS, 1979). In fact, that paper showed that Axioms 1 and 2 are exactly the properties that characterize the complete preorders $\succsim$ on $\mathscr{X}$ which admit a representation with uncertainty about future tastes. Formally, Kreps proves the following result:

Theorem 1 ((KREPS, 1979)). Let $\succsim$ be a binary relation on $\mathscr{X}$. Then, the following statements are equivalent:

(i) The relation $\succsim$ is a complete preorder that satisfies Monotonicity and Submodularity;

(ii) There exists a finite set $S$, a function $u: X \times S \rightarrow \mathbb{R}$ and a strictly increasing aggregator $V: \mathbb{R}^{S} \rightarrow \mathbb{R}$ such that, for every pair of menus $A$ and $B, A \succsim B$ if, and only if,

$$
V\left(\left(\max _{x \in A} u(x, s)\right)_{s \in S}\right) \geq V\left(\left(\max _{x \in B} u(x, s)\right)_{s \in S}\right)
$$

(iii) There exists a finite set $S$, a function $u: X \times S \rightarrow \mathbb{R}$ and a probability measure $\pi$ on $S$ such that, for every pair of menus $A$ and $B, A \succsim B$ if, and only if,

$$
\sum_{s \in S} \pi(s) \max _{x \in A} u(x, s) \geq \sum_{s \in S} \pi(s) \max _{x \in B} u(x, s)
$$

In the representation above, the DM is not sure about her future tastes. This is captured by the subjective state space $S$ and the state-dependent utility function $u$. Given this uncertainty, she anticipates the utility values she would obtain from a given menu in every possible future situation and aggregate them either by the use of a strictly increasing aggregator, (ii), or by the use of a subjective probability $\pi$ on $S,($ iii $)$.

1 See also (KOOPMANS, 1962).

2 Other papers, instead have worked with the notion of preference for freedom of choice. For that, see (PATTANAIK; XU, 1990), (SEN, 1991), (FOSTER, 1993) and (PUPPE, 1996). 
It turns out that the only property we have to add to Theorem 1 in order to characterize the relations which admit a weak categorization representation is the acyclicity of the relation $\triangleright$ induced by $\succsim$. Formally, consider the following postulate:

Axiom 3 (Acyclicity). For every $x_{1}, \ldots, x_{m} \in X$, if $x_{i} \triangleright x_{i+1}$ for $i=1, \ldots, m-1$, then it is not true that $x_{m} \triangleright x_{1}$.

The Acyclicity postulate imposes some consistency on the relation $\triangleright$. If we indeed interpret $\triangleright$ as a notion of revealed strict preferences, it is natural to rule out cycles as described in the postulate. We can now state the following result:

Theorem 2. Let $\succsim$ be a binary relation on $\mathscr{X}$. Then, the following statements are equivalent:

(i) The relation $\succsim$ is a complete preorder that satisfies Monotonicity, Submodularity and Acyclicity;

(ii) The relation $\succsim$ has a weak categorization representation;

(iii) The relation $\succsim$ has an additive weak categorization representation.

Theorem 2 shows that the axioms are necessary and sufficient for a relation $\succsim$ on $\mathscr{X}$ to be rationalized by a weak categorization representation. Moreover, it shows that, as it was the case with the representation in Theorem 1, additivity imposes no additional restrictions for weak categorization representations. This type of representation is essentially ordinal. In the next chapter we will see that this is no longer the case for categorization representations. However, it is important to mention that if $\succsim$ has a weak categorization representation $\langle u, \mathscr{C}, V\rangle$, it is not always the case that $V$ may be chosen to be additive in the sense of Definition 2 . The theorem only states that whenever $\succsim$ has a weak categorization representation $\langle u, \mathscr{C}, V\rangle$, it is always possible to find an additive weak categorization representation $\langle\hat{u}, \hat{\mathscr{C}}, \hat{\pi}\rangle$ of $\succsim$. There is no guarantee that such additive representation exists with $\mathscr{C}=\hat{\mathscr{C}}$ and $u=\hat{u}$, although we can always choose a $\hat{u}$ which is ordinally equivalent to $u$.

Note that, for any weak categorization representation $\langle u, \mathscr{C}, V\rangle$, if $x, y \in X$ are $\triangleright$-comparable, then $\{x, y\} \subseteq S$ for some $S \in \mathscr{C}$. This shows that $\triangleright$ imposes at least some restrictions on $\mathscr{C}$, but it does not guarantee the uniqueness of $\mathscr{C}$, for example. Thus, different weak categorizations may be chosen to represent the same relation $\succsim$. To illustrate that, consider the following example:

Example 1. Let $X=\{x, y, z\}$ and consider a complete preorder $\succsim$ on $\mathscr{X}$ given by $\{x, y, z\} \sim\{x, y\} \succ\{y, z\} \succ\{x, z\} \succ\{z\} \succ\{x\} \succ\{y\}$. Define $\left\langle u_{1}, \mathscr{C}_{1}, \pi_{1}\right\rangle$ and $\left\langle u_{2}, \mathscr{C}_{2}, \pi_{2}\right\rangle$ by $u_{1}(x)=1.1, u_{1}(y)=1.2, u_{1}(z)=1 ; \mathscr{C}_{1}=\{\{x, z\},\{y, z\}\} ; \pi_{1}(\{x, z\})=$ 
$0.6, \pi_{1}(\{y, z\})=0.4$ and $u_{2}(x)=1.2, u_{2}(y)=1.1, u_{2}(z)=1 ; \mathscr{C}_{2}=\{\{x, z\},\{y, z\},\{y\}\}$; $\pi_{2}(\{x, z\})=0.5, \pi_{2}(\{y, z\})=0.4, \pi_{2}(\{y\})=0.1$. It is clear that $\left\langle u_{1}, \mathscr{C}_{1}, \pi_{1}\right\rangle$ and $\left\langle u_{2}, \mathscr{C}_{2}, \pi_{2}\right\rangle$ are both additive weak categorization representations of $\succsim$.

The example above shows possible weak categorization representations of $\succsim$. Besides that, it is interesting to note that $x \triangleright z$ and $y \triangleright z$ even though $\{x, z\} \succ\{x\}$ and $\{y, z\} \succ\{y\}$. It is strictly better to add $z$ to both $\{x\}$ and $\{y\}$ but adding $z$ to $\{x, y\}$ is not. This shows that the value of $z$ relative to $x$ and $y$ depends not on the alternatives themselves, but rather on the menu they are inserted. In the next chapter, we will show that this will no longer happen if $\succsim$ has a categorization representation. 



\section{Categorization}

A categorization representation is a special case of a weak categorization representation, in which the categories form a partition of the set of alternatives. It turns out that such representation has very distinct properties when comparing menus inside a single category and when comparing menus that intersect several categories. For a categorization representation $\langle u, \mathscr{C}, V\rangle$, whenever two menus $A$ and $B$ are such that $(A \cup B) \subseteq S$ for some $S \in \mathscr{C}$, then $A \sim A \cup B$ or $B \sim A \cup B$. Therefore, flexibility has no value inside categories. However, whenever $A$ and $B$ are such that $A \subseteq S$ and $B \subseteq S^{\prime}$ for two distinct categories $S, S^{\prime} \in \mathscr{C}$, then $A \cup B \succ A$ and $A \cup B \succ B$. Thus, flexibility is always valuable when adding alternatives from distinct categories. Differently from the case of weak categorization, a categorization of $\succsim$ is unique and can be deduced from the preference itself.

It turns out that the differences between the weak categorization and categorization models all stem from the properties of the relation $\triangleright$. In particular, in order to characterize the relations $\succsim$ that have a categorization representation we need to impose some restriction on the way $\triangleright$ relates to the DM's preferences over menus of two or less elements. Formally, consider the following postulate:

Axiom 4 (Compatibility). The relation $\triangleright$ is asymmetric and, for every $x, y, z \in X$, if $x \triangleright y$, then $\{x, z\} \succ\{x\},\{z\}$ if, and only if, $\{y, z\} \succ\{y\},\{z\}$.

First of all, Compatibility imposes that $\triangleright$ is entirely captured by the DM's preferences over menus of two or less options, in the sense that $x \triangleright y$ if, and only if, $x \neq y$ and $\{x\} \sim\{x, y\}$. This implies that, whenever $x \in X$ and $A \in \mathscr{X}$ are such that $\{x, y\} \succ\{y\}$ for every $y \in A$, then $A \cup\{x\} \succ A$ (put it differently, whenever $A \cup\{x\} \sim A$ and $x \notin A$, then there exists $y \in A$ such that $\{x, y\} \sim\{y\})$. Thus, the DM is indifferent between choosing a menu and the menu containing only its $\triangleright$-maximal elements. Note that this was not the case for weak categorization representation as seen in Example 1. Moreover, the axiom imposes a comparability transitivity of $\triangleright$, that is, whenever two alternatives in $X$ are $\triangleright$-comparable to a mutual alternative, then they are also $\triangleright$-comparable. Note that, in Example 1, we have $x \triangleright z$ and $y \triangleright z$ but $x$ and $y$ are not $\triangleright$-comparable. Given this additional condition, we can now prove the following result:

Theorem 3. Let $\succsim$ be a binary relation on $\mathscr{X}$. Then, the following statements are equivalent:

(i) The relation $\succsim$ is a complete preorder that satisfies Monotonicity, Submodularity and Compatibility; 
(ii) The relation $\succsim$ has a categorization representation.

Theorem 3 shows that Compatibility is a sufficient additional condition for a relation on $\mathscr{X}$ to have a categorization representation. However, differently from the case of weak categorization, for categorization representations additivity imposes an additional restriction. Too illustrate that, consider the following example:

Example 2. Let $X=\{x, y, z\}$ and consider a complete preorder $\succsim$ on $\mathscr{X}$ given by $\{x, y, z\} \succ\{y, z\} \succ\{x, y\} \succ\{x, z\} \succ\{x\} \succ\{y\} \succ\{z\}$. It is clear that $\succsim$ admits a categorization representation $\langle u, \mathscr{C}, V\rangle$ with $\mathscr{C}=\{\{x\},\{y\},\{z\}\}$. However, for any $u$ : $X \rightarrow \mathbb{R}_{++}$and probability measure $\pi$ on $\mathscr{C}, \pi(\{x\}) u(x)>\pi(\{y\}) u(y)$ implies $\pi(\{x\}) u(x)+$ $\pi(\{z\}) u(z)>\pi(\{y\}) u(y)+\pi(\{z\}) u(z)$. Consequently, $\succsim$ does not admit an additive categorization representation. However, it does admits an additive weak categorization representation $\langle u, \mathscr{C}, \pi\rangle$ where $u(x)=1, u(y)=2, u(z)=1.5 ; \mathscr{C}=\{\{x, y\},\{x, z\},\{x\}\}$; $\pi(\{x, y\})=\pi(\{x, z\})=0.45$ and $\pi(\{x\})=0.1$.

In order to understand why additivity is restrictive when we deal with categorization representations, suppose $\succsim$ is a complete preorder on $\mathscr{X}$ that has a categorization representation $\langle u, \mathscr{C}, V\rangle$. Enumerate the categories in $\mathscr{C}$ so that it can be written as $\mathscr{C}=\left\{S_{1}, \ldots, S_{n}\right\}$. Let $Y:=\left(\left(S_{1} \cup\{0\}\right) \times \ldots \times\left(S_{n} \cup\{0\}\right)\right)$, where 0 represents an element that does not belong to $X$. Now for each $A \in \mathscr{X}$, let $\xi^{A} \in Y$ be such that $\xi_{i}^{A}$ is the element of $S_{i}$ that maximizes $u$ in $A \cap S_{i}$, with the convention that $\xi_{i}^{A}=0$ if $A \cap S_{i}=\emptyset$. Finally, define a binary relation $\succcurlyeq \subseteq Y \times Y$ by $\xi \succcurlyeq \zeta$ if, and only if, there exist $A, B \in \mathscr{X}$ with $\xi=\xi^{A}, \zeta=\xi^{B}$ and $A \succsim B$. It is easy to see that $\succcurlyeq$ is a well-defined preorder and, for every pair of menus $A$ and $B$ in $\mathscr{X}, A \succsim B$ if, and only if, $\xi^{A} \succcurlyeq \xi^{B}$. Moreover, it turns out that $\succsim$ admits an additive categorization representation if, and only if, $\succcurlyeq$ admits an additive representation. Since it is well-known that for a preorder on a finite cartesian space like $Y$ we need an additional condition to guarantee it has an additive representation, it is clear that we need an additional postulate in order to obtain an additive categorization representation for $\succsim$. We now adapt the standard condition used in the cartesian preferences setup to the setup here, in order to obtain an additive version of the representation in Theorem 3. First we need the following definition:

Definition 4. Given a finite sequence of menus $\left(A^{m}\right)$ and an alternative $x \in X$, let $n\left(\left(A^{m}\right), x\right)$ be the number of elements of the sequence $\left(A^{m}\right)$ in which $x$ is $\triangleright$-maximal.

Given the definition above, we can now state the following postulate:

Axiom 5 (Finite Cancellation). Let $\left(A^{m}\right)$ and $\left(B^{m}\right)$ be any two finite sequences of menus with the same length. If $n\left(\left(A^{m}\right), x\right)=n\left(\left(B^{m}\right), x\right)$ for every $x \in X$, then it cannot be the case that $A^{m} \succsim B^{m}$ for every $m$ and $A^{m} \succ B^{m}$ for some $m$. 
The condition above is a translation of the finite cancellation postulate that characterizes additive representation of cartesian preferences. (see, for example, (WAKKER, 2013)). The idea is that, whenever $\succsim$ has an additive representation, then given finite sequences $\left(A^{m}\right)$ and $\left(B^{m}\right)$ such that its $\triangleright$-maximal elements are equally distributed and $A^{m} \succsim B^{m}$ for every $m$, then the cancellation property of real numbers imposes that $A^{m} \sim B^{m}$ for every $m$. Given this condition, we can now prove the following result:

Theorem 4. Let $\succsim$ be a binary relation on $\mathscr{X}$. Then, the following statements are equivalent:

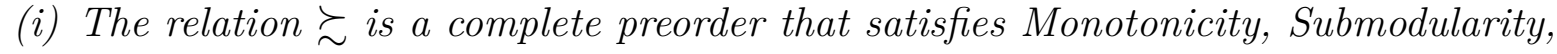
Compatibility and Finite Cancellation;

(ii) The relation $\succsim$ has an additive categorization representation.

With Theorem 4, we conclude our main representation theorems. We now turn to two extreme cases of categorization, meaning the cases in which $\mathscr{C}$ is either the coarsest or the finest partition of $X$. With that in mind, let's call $\mathscr{C}$ an extreme categorization of $X$ if $\mathscr{C}=\{X\}$, or $\mathscr{C}=\{\{x\}: x \in X\}$. In the first case, all elements of $X$ belong to the same category, while in the second each single element of $X$ forms a different category, It turns out that extreme categorizations correspond to the extreme cases of preferences for flexibility and are characterized by the extreme degrees of comparability of the relation $\triangleright$. A slightly more surprising result is the fact that a single strengthening of Compatibility characterizes the relations $\succsim$ that admit an extreme categorization representation. Formally, consider the following postulate:

Axiom 6 (Strong Compatibility). The relation $\triangleright$ is asymmetric and, for every $x, y, z \in X$, if $x \triangleright y$, then neither $\{x, z\} \succ\{x\},\{z\}$ nor $\{y, z\} \succ\{y\},\{z\}$.

We can now state the following result:

Theorem 5. Let $\succsim$ be a binary relation on $\mathscr{X}$. Then, the following statements are equivalent:

(i) The relation $\succsim$ is a complete preorder that satisfies Monotonicity, Submodularity and Strong Compatibility;

(ii) The relation $\succsim$ has an extreme categorization representation.

Theorem 5 has the following corollaries:

Corollary 1. Let $\succsim$ be a binary relation on $\mathscr{X}$ such that $\triangleright=\emptyset$. Then, the following statements are equivalent: 
(i) The relation $\succsim$ is a complete preorder that satisfies Monotonicity;

(ii) The relation $\succsim$ has a categorization representation in which $\mathscr{C}=\{\{x\}: x \in X\}$.

Corollary 2. Let $\succsim$ be a binary relation on $\mathscr{X}$ such that $\triangleright \neq \emptyset$. Then, the following statements are equivalent:

(i) The relation $\succsim$ is a complete preorder that satisfies Monotonicity, Submodularity and Strong Compatibility;

(ii) The relation $\succsim$ has a categorization representation in which $\mathscr{C}=\{X\}$. 


\section{Preference for Flexibility and Categoriza- tion}

Over this thesis, all the analysis was based on the relation $\triangleright$, which is captured in terms of preference for flexibility. In Kreps' representation, as discussed in chapter 3, the preference for flexibility is due to an uncertainty about the DM's future tastes. In the representation by categorization, it is due to an uncertainty about the relevance of alternatives. Thus, conceptually, the connection between the two representations is given by the uncertainty about future choices.

It turns out that we can directly relate the representation in (KREPS, 1979) with the idea of categorization. In fact, it is well known that if $\succsim$ has a representation as in Theorem 1, then it admits a representation in the style of the additive weak categorization representation but without the requirement that $u$ be injective. ${ }^{1}$ That is, the only implication of Acyclicity is the injectivity of $u$. However, without the requirement that the function $u$ be injective, this function has very limited behavioral content, especially when $\triangleright$ indeed has cycles. To illustrate that, let $X:=\left\{x_{1}, \ldots, x_{n}\right\}$ with $n>4$ and suppose there are two states of nature $s_{1}, s_{2}$ in which the DM's ex-post preferences are $u\left(x_{i}, s_{1}\right)>u\left(x_{i+1}, s_{1}\right)$ and $u\left(x_{i+1}, s_{2}\right)>u\left(x_{i}, s_{2}\right)$ for $i=1, \ldots, n-1$. Note that, for any $x_{i}$ and $x_{j}$ with $n>j>i>1$, we have $\left\{x_{1}, x_{i}, x_{j}\right\} \sim\left\{x_{1}, x_{j}\right\} \succ\left\{x_{1}, x_{i}\right\} \succ\left\{x_{1}\right\}$ and $\left\{x_{n}, x_{i}, x_{j}\right\} \sim\left\{x_{n}, x_{i}\right\} \succ\left\{x_{n}, x_{j}\right\} \succ\left\{x_{n}\right\}$. Thus, $x_{i} \triangleright x_{j}$ and $x_{j} \triangleright x_{i}$. This implies that for any representation of $\succsim$ in the format of weak categorization, we must have $u\left(x_{i}\right)=u\left(x_{j}\right)$ for every $i, j \in\{2, \ldots, n-1\}$. In turn, this implies that, for every menu $A \subseteq\left\{x_{2}, \ldots, x_{n-1}\right\}$, all alternatives in $A$ are possible future choices if we represent this preferences in a weak categorization format. When $u$ is injective, it necessarily agrees with the relation $\triangleright$ and, consequently, $u$ has some behavioral content.

Within the context of preference for flexibility, (BARBERÀ; GRODAL, 2011) also present a similar additive representation as the one developed here. Although the representation is similar, they give a different interpretation. They interpret it as working in an environment where flexibility is desirable as consequence of an uncertainty about the availability of the alternatives in the act of choice. Hence, the DM's uncertainty about future choices is given only by external constraints, while we favor the interpretation of a self-imposed constraint. Another difference is that they consider only linear orders $\succsim$ on $\mathscr{X}$. In such case, we have seen in the previous chapter that $\triangleright=\emptyset$ and thus $\succsim$ only needs to satisfy Monotonicity to admit an additive weak categorization representation since it

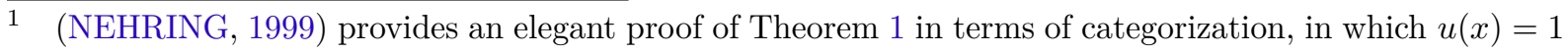
for every $x \in X$. 
admits a categorization representation by Corollary 1. Consequently, our representation requires a deeper analysis since all subtle aspects of it rely on the cases in which flexibility stops being valuable. 


\section{Literature Discussion}

Recently, several papers have developed models of choice that consider categorization as part of the decision making process, both within a setup of deterministic or stochastic choices. As we will show next, some of these works have a similar framework as ours. However, all of them develop models of choosing alternatives from the menus, while our model describes a choice of menus.

In the setup of deterministic choice, (FURTADO; NASCIMENTO; RIELLA, 2017) develop a model in which the DM is endowed with a preference (possibly incomplete) over the alternatives and categorize the set of alternatives with the condition that her preference is complete within each category. Facing a choice problem, the DM's choice is simply the union of the best available alternatives from each category. As we have mentioned in the Introduction, their analysis also rely on the cases of weak categorization and categorization of $X$. Interestingly, they characterize both representations based on a revealed preference relation which requires similar postulates as Acyclicity, for weak categorization representation, and Compatibility, for categorization representation.

(BARBOS, 2010) and (MANZINI; MARIOTTI, 2012) also characterize deterministic choice models in the presence of categorization. Differently from (FURTADO; NASCIMENTO; RIELLA, 2017), both works assume not only a preference over the set of alternatives but also a preference over a set of categories. In (BARBOS, 2010), each choice problem has an exogenously given categorization and the DM chooses the best alternatives from the most preferred categories. In (MANZINI; MARIOTTI, 2012), the DM first eliminates the alternatives that belong to dominated categories that are contained in the choice problem and then chooses the best of the remaining alternatives. Although both works characterize a two-stage model of choice with categorization, as ours, in their models the choice of a category is given by the preference over them. In our model, the

choice of a category is random, even though $\succsim$ induces, in some sense, a preference over the categories.

Within the setup of stochastic choice, (AGUIAR, 2016) and (ZHANG, 2016) formulate distinct models of choice with categorization. The former develops a model of stochastic choices in which the DM has an uncertainty about which category he will constrain its choice problem to. The probability of choosing an alternative is given by the probability of drawing a category from which this alternative is the best available option. The latter develops a model in which the DM partitions the set of alternatives $X$ into categories and possesses a "search order" over the categories. She then considers categories sequentially until making a choice. 



\section{Conclusion}

In the present thesis, we developed a theory of preference for flexibility in a setup of categorization. We extended the work of (FURTADO; NASCIMENTO; RIELLA, 2017) to a setup of preference over menus. Imposing a similar acyclicity condition, we obtained both ordinal and additive representations that make use of a weak categorization of the space of alternatives. For the case where categories are pairwise disjoint, imposing a postulate of compatibility of the revealed preference, we obtained the ordinal representation, where this was not enough to obtain the additive one. For this, we adapted the condition of additivity for cartesian preferences and showed that this is also the necessary and sufficient condition for additivity in our setup. 

Appendix 



\section{APPENDIX A - Proofs}

\section{A.1 Proof of Theorem 2}

Is is clear that an additive weak categorization representation is a special case of a weak categorization representation, so it is immediate that (iii) implies (ii). It is also easy to check that $\succsim$ is a complete preorder that satisfies Monotonicity, Submodularity and Acyclicity whenever it has a weak categorization representation. So we will show only that (i) implies (iii).

For that, suppose $\succsim$ is a complete preorder on $\mathscr{X}$ that satisfies Monotonicity, Submodularity and Acyclicity. Let $\mathscr{C}$ be any nonempty subset of $2^{X}$. Define a function $U: X \times \mathscr{C} \rightarrow \mathbb{R}$ by $U(x, S):=-1$ if $x \in S$ and $U(x, S):=0$, otherwise. Now, for each $A \in 2^{X}$, define a function $\sigma_{A}: \mathscr{C} \rightarrow \mathbb{R}$ by $\sigma_{A}(S):=\max _{x \in A} U(x, S)$ if $A \in \mathscr{X}$, and $\sigma_{\emptyset}(\emptyset)=-1$ and $\sigma_{A}(\emptyset):=0$ for every $A \neq \emptyset$. Note that $\sigma_{A}(S)=-1$ if $A \subseteq S$ and $\sigma_{A}(S)=0$ otherwise. In what follows we will make use of the following lemma:

Lemma 1. Let $\mathscr{C}$ be a nonempty subset of $2^{X}$ and let the vectors $\sigma_{A}$ be defined as above for every $A \in \mathscr{C}$. Then, $\left\{\sigma_{A}: A \in \mathscr{C}\right\}$ is a base for $\mathbb{R}^{\mathscr{C}}$.

Proof. Enumerate the elements of $\mathscr{C}$ in non-increasing order of cardinality, that is, such that $\left|S_{1}\right| \geq\left|S_{2}\right| \geq \cdots \geq\left|S_{|\mathscr{C}|}\right|$ and denote by $\Sigma$ the $|\mathscr{C}| \times|\mathscr{C}|$ matrix such that $\left\{\sigma_{A}: A \in \mathscr{C}\right\}$ are its columns and ordered the same way $\mathscr{C}$ is. Notice that, for any $A=S_{i}$, we have $\sigma_{A}\left(S_{i}\right)=-1$ and $\sigma_{A}\left(S_{j}\right)=0$ if $j>i$. Therefore, we obtain an upper triangular matrix whose main diagonal elements are all equal to -1 . Hence $A$ is a nonsingular matrix and its columns are a basis for $\mathbb{R}^{\mathscr{C}}$.

Now, fix any function $v: X \rightarrow \mathbb{R}$. For each $\varepsilon>0$ and $A \in 2^{X}$, define the function $\gamma_{A}^{\varepsilon}: \mathscr{C} \rightarrow \mathbb{R}$ by $\gamma_{A}^{\varepsilon}(S)=\varepsilon \max v(A \backslash S)$ if $A \backslash S \neq \emptyset$, and $\gamma_{A}^{\varepsilon}(S)=-1$ otherwise. Lemma 1 has the following corollary:

Corollary 1. Let $\mathscr{C}$ be a nonempty subset of $2^{X}$ and fix $v: X \rightarrow \mathbb{R}$. Let the vectors $\gamma_{A}^{\varepsilon}$ be defined as above, for every $A \in \mathscr{C}$ and $\varepsilon>0$. Then, there exists $\varepsilon>0$ such that $\left\{\gamma_{A}^{\varepsilon}: A \in \mathscr{C}\right\}$ is a base for $\mathbb{R}^{\mathscr{C}}$.

Proof. For each $\varepsilon>0$, let $\Gamma_{\varepsilon}$ be the $|\mathscr{C}| \times|\mathscr{C}|$ matrix such that $\left\{\gamma_{A}^{\varepsilon}: A \in \mathscr{C}\right\}$ are its columns and ordered the same way as in Lemma 1. Since $\lim _{\varepsilon \rightarrow 0} \gamma_{A}^{\varepsilon}(S)=\sigma_{A}(S)$ for every $A$ and $S$ in $\mathscr{C}$ and since the determinant is a continuous operator, Lemma 1 implies that, for $\varepsilon$ small enough, $\Gamma_{\varepsilon}$ is nonsingular. 
Now define a self-map $f$ on $\mathscr{X}$ by $f(A):=\bigcup\{B \in \mathscr{X}: A \subseteq B$ and $A \sim B\}$ for every $A \in \mathscr{X}$. (KREPS, 1979) showed that $f(A) \sim B$ for every $A, B \in \mathscr{X}$ with $A \subseteq B \subseteq f(A)$ and that $A \subseteq f(C)$ if and only if $f(A) \subseteq f(C)$ for every $A, C \in \mathscr{X}$. Define $\mathscr{C}:=f(\mathscr{X}) \cup\{\emptyset\}$.

Let $\left\{I_{1}, I_{2}, \ldots, I_{n}\right\}$ be the indifference classes of $\succsim$ ordered such that, for every $A \in I_{i}$ and $B \in I_{j}, A \succsim B$ if and only if $i \leq j$, and define $V: 2^{X} \rightarrow \mathbb{R}$ by $V(A):=-|\mathscr{C}|^{i-1}$ for every $A \in \mathscr{X}$, where $i$ is such that $A \in I_{i}$, and $V(\emptyset)=-|\mathscr{C}|^{n}$. Clearly, $\left.V\right|_{\mathscr{X}}$ represents $\succsim$. Define the functions $\sigma_{A}: \mathscr{C} \rightarrow \mathbb{R}$ as previously, for every $A \in 2^{X}$ and notice that $\sigma_{A}=\sigma_{f(A)}$. Lemma 1 guarantees that $\left\{\sigma_{A}: A \in \mathscr{C}\right\}$ is a base for $\mathbb{R}^{\mathscr{C}}$. Therefore, there is a unique vector $\bar{\pi} \in \mathbb{R}^{\mathscr{C}}$ such that $\sum_{S \in \mathscr{C}} \bar{\pi}(S) \sigma_{A}(S)=V(A)$. We will show that $\bar{\pi} \in \mathbb{R}_{++}^{\mathscr{C}}$. Note first that $\bar{\pi}(X)=1$. Suppose now that $A \in \mathscr{C} \backslash\{X\}$ is such that $\bar{\pi}(S)>0$ for all $S \in \mathscr{C}$ with $A \subseteq S$ and $A \neq S$. Let $I_{i}$ be the indifference class of $A$ and notice that $S \in I_{j}$ with $j<i$ for every $S$ as above. Finally, we have

$$
\begin{aligned}
V(A) & =-\bar{\pi}(A)-\sum_{\{S \in \mathscr{C}: A \subseteq S \text { and } A \neq S\}} \bar{\pi}(S) \\
& \geq-\bar{\pi}(A)-\sum_{\{S \in \mathscr{C}: A \subseteq S \text { and }} \sum_{A \neq S\}} \overline{\{T \in \mathscr{C}: S \subseteq T\}}(T) \\
& =-\bar{\pi}(A)+\sum_{\{S \in \mathscr{C}: A \subseteq S \text { and } A \neq S\}} V(S) \\
& >-\bar{\pi}(A)+|\mathscr{C}| \cdot V\left(I_{i-1}\right) \\
& =-\bar{\pi}(A)+V(A),
\end{aligned}
$$

which implies that $\bar{\pi}(A)>0$. We conclude that $\bar{\pi} \in \mathbb{R}_{++}^{\mathscr{C}}$. Now, we can move to the representation we desire. Define the binary relation $\triangleright$ on $X$ as in Definition 3. Since it is acyclic by hypothesis, the transitive closure of $\triangleright$ is a strict partial order, which, by the Szpilrajn Theorem, can be extended to a linear order $\succcurlyeq$. Take any utility function $v: X \rightarrow \mathbb{R}_{++}$that represents $\succcurlyeq$. Notice that, for any scalar $\varepsilon>0$, $\varepsilon v$ still represents $\succcurlyeq$. For any $\varepsilon>0$, define the function $\gamma_{A}^{\varepsilon}: \mathscr{C} \rightarrow \mathbb{R}$ as in Corollary 1 , for every $A \in 2^{X}$. We claim that $\gamma_{A}^{\varepsilon}=\gamma_{f(A)}^{\varepsilon}$ for every $A \in \mathscr{X}$. To see that, fix any $A \in \mathscr{X}$ and $S \in \mathscr{C}$. Since we have $A \subseteq S$ if and only if $f(A) \subseteq S$, it is immediate that $\gamma_{A}^{\varepsilon}(S)=\gamma_{f(A)}^{\varepsilon}(S)=-1$ if $A \subseteq S$. For the case in which $A \backslash S \neq \emptyset$, we need the following claim:

Claim 1. For every $A \in \mathscr{X}, S \in \mathscr{C}$ and $y \in(f(A) \backslash A) \backslash S$, there exists $x \in A \backslash S$ such that $x \triangleright y$.

Proof. Fix any $A \in \mathscr{X}$ and $S \in \mathscr{C}$ such that $(f(A) \backslash A) \backslash S \neq \emptyset$ and pick any $y \in$ $(f(A) \backslash A) \backslash S$. Notice that we must have $A \cup\{y\} \sim A$ and $S \cup\{y\} \succ S$. If $A \cap S=\emptyset$, then $A \backslash S=A$ and it's easy to check that there exists $x \in A$ such that $x \triangleright y$. If $A \cap S \neq \emptyset$, 
Submodularity implies that $(A \cap S) \cup\{y\} \succ A \cap S$. So, there must exist $x \in A \backslash S$ such that $B \cup\{y\} \succ B$ but $B \cup\{x, y\} \sim B \cup\{x\}$ for some $B \in \mathscr{X}$ with $A \cap S \subseteq B \subseteq A$.

By definition, we have $u(x)>u(y)$ whenever $x \triangleright y$. So the claim above implies that $\max u(A \backslash S)=\max u(f(A) \backslash S)$ even when $A \backslash S \neq \emptyset$. We conclude that $\gamma_{A}^{\varepsilon}=\gamma_{f(A)}^{\varepsilon}$ for every $A \in \mathscr{X}$. Let $V: 2^{X} \rightarrow \mathbb{R}$ be defined as above. By Corollary 1, for $\varepsilon>0$ small enough we have that $\left\{\gamma_{A}^{\varepsilon}: A \in \mathscr{C}\right\}$ is a base for $\mathbb{R}^{\mathscr{C}}$. This implies that there exists a unique $\pi^{\varepsilon} \in \mathbb{R}^{\mathscr{C}}$ such that $\sum_{S \in \mathscr{C}} \pi^{\varepsilon}(S) \gamma_{A}^{\varepsilon}(S)=V(A)$ for every $A \in \mathscr{C}$.

It remains to show that $\pi^{\varepsilon} \in \mathbb{R}_{++}^{\mathscr{C}}$ for $\varepsilon>0$ small enough. To see that, let's think of the restriction of $V$ to $\mathscr{C},\left.V\right|_{\mathscr{C}}$, as a vector in $\mathbb{R}^{\mathscr{C}}$. We have shown that, for $\varepsilon>0$ small enough, we have $\pi^{\varepsilon}=\left.\left(\Gamma_{\varepsilon}^{T}\right)^{-1} V\right|_{\mathscr{C}}$ and $\bar{\pi}=\left.\left(\Sigma^{T}\right)^{-1} V\right|_{\mathscr{C}}$. Since $\lim _{\varepsilon \rightarrow 0} \Gamma_{\varepsilon}=\Sigma$ and $\bar{\pi} \in \mathbb{R}_{++}^{\mathscr{C}}$, we get that $\pi^{\varepsilon} \in \mathbb{R}_{++}^{\mathscr{C}}$ for $\varepsilon>0$ small enough by continuity.

Finally, fix $\varepsilon>0$ small enough so that $\pi^{\varepsilon}$ is well-defined and $\pi^{\varepsilon} \in \mathbb{R}_{++}^{\mathscr{C}}$. Define $\hat{\mathscr{C}}$ by $\hat{\mathscr{C}}:=\{S \in \mathscr{X}: X \backslash S \in \mathscr{C}\}$. Define $\pi \in \mathbb{R}_{++}^{\hat{\mathscr{C}}}$ by $\pi(S):=\pi^{\varepsilon}(X \backslash S)\left(\sum_{\hat{S} \in \hat{\mathscr{C}}} \pi^{\varepsilon}(X \backslash \hat{S})\right)^{-1}$ for every $S \in \hat{\mathscr{C}}$. Define $u: X \rightarrow \mathbb{R}_{++}$by $u(x):=1+\varepsilon v(x)$ for every $x \in X$. Note that, for every $A \in \mathscr{X}$,

$$
\sum_{S \in \hat{\mathscr{C}}} \pi(S) \max _{x \in A \cap S} u(x)=\left[\left(V(A)+\pi^{\varepsilon}(X)\right)\left(\sum_{\hat{S} \in \hat{\mathscr{C}}} \pi^{\varepsilon}(X \backslash \hat{S})\right)^{-1}\right]+1
$$

with the convention that $\max u(\emptyset)=0$. This shows that $\langle u, \hat{\mathscr{C}}, \pi\rangle$ is an additive weak categorization representation of $\succsim$.

\section{A.2 Proof of Theorem 3}

Suppose first that $\succsim$ is a relation on $\mathscr{X}$ that has a categorization representation $\langle u, \mathscr{C}, V\rangle$. By Theorem 2 we already know that $\succsim$ is a complete preorder and satisfies Monotonicity and Submodularity. We only need to show that $\succsim$ satisfies Compatibility. For that, fix any $x, y \in X$ such that $x \triangleright y$. This can only happen if $\{x, y\} \subseteq S$ for some $S \in \mathscr{C}$ and $u(x)>u(y)$. But then it is clear that we cannot have $y \triangleright x$. This shows that $\triangleright$ is asymmetric. Again, suppose that $x \triangleright y$, fix any $z \in X$ and let $S \in \mathscr{C}$ be such that $\{x, y\} \subseteq S$. Since $\mathscr{C}$ is a partition of $X$, it is clear that $\{x, z\} \succ\{x\},\{z\}$ happens if, and only if, $z \notin S$, which happens if, and only if, $\{y, z\} \succ\{y\},\{z\}$. We conclude that $\succsim$ satisfies Compatibility.

Conversely, suppose $\succsim$ is a complete preorder on $\mathscr{X}$ that satisfies Monotonicity, Submodularity and Compatibility. We first need the following claim:

Claim 1. For every $x, y \in X, x \triangleright y$ if, and only if, $x \neq y$ and $\{x\} \sim\{x, y\}$. Moreover, $\triangleright$ is transitive. 
Proof. By definition, $x \triangleright y$ whenever $x \neq y$ and $\{x\} \sim\{x, y\}$. Conversely, suppose $x \triangleright y$. Applying the Compatibility postulate with $z=x$, we see that we must have $\{x\} \sim\{x, y\}$ or $\{y\} \sim\{x, y\}$. Since $\triangleright$ is asymmetric and $\{y\} \sim\{x, y\}$ would imply $y \triangleright x$, we must have $\{x\} \sim\{x, y\}$. Let's now show that $\triangleright$ is transitive. For that, suppose $x \triangleright y$ and $y \triangleright z$. We have already seen that this implies that $\{x\} \sim\{x, y\}$ and $\{y\} \sim\{y, z\}$. Moreover, since $\triangleright$ is asymmetric, we must also have $\{x, y\} \succ\{y\}$ and $\{y, z\} \succ\{z\}$. Now Submodularity implies that $\{x, y, z\} \sim\{x, y\} \sim\{x, z\} \sim\{x\}$. Since $\triangleright$ is asymmetric, we cannot have $x=z$, which implies that $x \triangleright z$. This shows that $\triangleright$ is transitive.

Now define a binary relation $I$ on $X$ by $x I y$ if, and only if, $\{x\} \sim\{x, y\}$ or $\{y\} \sim\{x, y\}$. We need the following claim:

Claim 2. The relation $I$ is an equivalence relation.

Proof. The relation $I$ is clearly reflexive and symmetric. It remains to show it is transitive. Suppose, then, that $x I y$ and $y I z$. If $x=y$ or $y=z$, there is nothing to prove, so suppose $x \neq y$ and $y \neq z$. In this case, $x \triangleright y$ or $y \triangleright x$ and it is not true that $\{y, z\} \succ\{y\},\{z\}$. Now Compatibility implies that we cannot have $\{x, z\} \succ\{x\},\{z\}$, which implies that $\{x\} \sim\{x, z\}$ or $\{z\} \sim\{x, z\}$ and, consequently, $x I z$.

Now, for each $x \in X$, define $S_{x}:=\{y \in X: x I y\}$ and let $\mathscr{C}:=\left\{S_{x}: x \in X\right\}$. Since $I$ is an equivalence relation, it is well-known that $\mathscr{C}$ is a partition of $X$. Notice that $x$ and $y$ are $\triangleright$-comparable if and only if $\{x, y\} \subseteq S$ for some $S \in \mathscr{C}$. Since $\triangleright$ is a strict partial order, it can be extended to a linear order $\succcurlyeq$. Let $u: X \rightarrow \mathbb{R}_{++}$be an utility representation of $\succcurlyeq$ and define, for each $A \in \mathscr{X}, \delta_{A}: \mathscr{C} \rightarrow \mathbb{R}$ by $\delta_{A}(S):=\max u(A \cap S)$ if $A \cap S \neq \emptyset$ and $\delta_{A}(S):=0$ otherwise. We note that, for any two menus $A, B \in \mathscr{X}, \delta_{A}=\delta_{B}$ if, and only if, $\operatorname{MAX}(A, \triangleright)=M A X(B, \triangleright)$. Moreover, a simple inductive argument based on the definition of $\triangleright$ shows that $A \sim \operatorname{MAX}(A, \triangleright)$ for every $A \in \mathscr{X}$. This allows us to find a function $V: \mathbb{R}^{\mathscr{C}} \rightarrow \mathbb{R}$ such that $V\left(\delta_{A}\right) \geq V\left(\delta_{B}\right)$ if, and only if, $A \succsim B$, for every $A, B \in \mathscr{X}$. To show that $V$ is strictly increasing on its relevant domain, define, for each menu $A \in \mathscr{X}$, the set $L_{\triangleright}(A):=A \cup\{y \in X: x \triangleright y$ for some $x \in A\}$. Now suppose $A, B \in \mathscr{X}$ are such that $\delta_{A} \geq \delta_{B}$ and $\delta_{A} \neq \delta_{B}$. This happens if, and only if, $L_{\triangleright}(B)$ is a proper subset of $L_{\triangleright}(A)$. Moreover, a simple inductive argument shows that $L_{\triangleright}(B) \cup\{x\} \succ L_{\triangleright}(B)$ for every $x \in X \backslash L_{\triangleright}(B)$. But then we have $A \sim L_{\triangleright}(A) \succ L_{\triangleright}(B) \sim B$, which implies that $V\left(\delta_{A}\right)>V\left(\delta_{B}\right)$. This shows that $V$ is strictly increasing on its relevant domain. It is easy to extend $V$ to a stricly increasing function on $\mathbb{R}^{\mathscr{C}}$. Putting everything together, we get that $\langle u, \mathscr{C}, V\rangle$ is a categorization representation of $\succsim$. 


\section{A.3 Proof of Theorem 4}

Suppose first that $\succsim$ is a relation on $\mathscr{X}$ that has an additive categorization representation $\langle u, \mathscr{C}, \pi\rangle$. By Theorem 3 , we already know that $\succsim$ is a complete preorder that satisfies Monotonicity, Submodularity and Compatibility. To show that it satisfies Finite Cancellation, fix any two finite sequences $\left(A^{m}\right)$ and $\left(B^{m}\right)$ in $\mathscr{X}$ such that $n\left(\left(A^{m}\right), x\right)=$ $n\left(\left(B^{m}\right), x\right)$ for every $x \in X$ and suppose $A^{m} \succsim B^{m}$ for every $m$ and $A^{m} \succ B^{m}$ for some $m$. Then, we must have $\sum \sum_{S \in \mathscr{C}} \pi(S) \max u\left(A^{m} \cap S\right)>\sum \sum_{S \in \mathscr{C}} \pi(S) \max u\left(B^{m} \cap S\right)$. However, since $n\left(\left(A^{m}\right), x\right)=n\left(\left(B^{m}\right), x\right)$ for every $x \in X$, every term above occurs the same number of times on both sides, so that the strict inequality above is not possible. We conclude that $\succsim$ satisfies Finite Cancellation.

Conversely, suppose that $\succsim$ is a complete preorder on $\mathscr{X}$ that satisfies Monotonicity, Submodularity, Compatibility and Finite Cancellation. By Theorem 3, we know that $\succsim$ has a categorization representation $\langle u, \mathscr{C}, V\rangle$. For every menu $A \in \mathscr{X}$, define $\delta_{A} \in \mathbb{R}^{\mathscr{C}}$ by $\delta_{A}(S):=\max u(A \cap S)$, with the convention that $\max u(A \cap S)=0$ when $A \cap S=\emptyset$. Now let $Y:=\left\{\delta_{A}: A \in \mathscr{X}\right\}$. Let $\hat{\succsim}$ be the complete preorder $V$ induces on $Y$. It is easy to see that the fact that $\succsim$ satisfies Finite Cancellation implies that $\hat{\succsim}$ satisfies the Finite Cancellation axiom for cartesian preferences (see (WAKKER, 2013), page 33, for example). This now implies that there exist functions $w_{S}: u(S) \cup\{0\} \rightarrow \mathbb{R}$ for every $S \in \mathscr{C}$ such that, for any pair of menus $A$ and $B$,

$$
\delta_{A} \hat{\succsim} \delta_{B} \Leftrightarrow \sum_{S \in \mathscr{C}} w_{S}\left(\delta_{A}(S)\right) \geq \sum_{S \in \mathscr{C}} w_{S}\left(\delta_{B}(S)\right)
$$

Without loss of generality, we can assume that $w_{S}(0)=0$ for every $S \in \mathscr{C}$. Since $\hat{\succsim}$ is strictly increasing and $Y$ has a cartesian product structure, we must have that $w_{S}(u(x))>0$ for every $S \in \mathscr{C}$ and $x \in S$. Moreover, for any $S \in \mathscr{C}$ and any $x, y \in S$, we must have $w_{S}(u(x)) \geq w_{S}(u(y))$ if, and only if, $u(x) \geq u(y)$. But then, for every pair of menus $A$ and $B$,

$$
\begin{aligned}
A \succsim B & \Leftrightarrow & \delta_{A} \hat{\succsim} \delta_{B} \\
& \Leftrightarrow & \frac{1}{|\mathscr{C}|} \sum_{S \in \mathscr{C}} w_{S}\left(\delta_{A}(S)\right) \geq \frac{1}{|\mathscr{C}|} \sum_{S \in \mathscr{C}} w_{S}\left(\delta_{B}(S)\right) \\
& \Leftrightarrow & \frac{1}{|\mathscr{C}|} \sum_{S \in \mathscr{C}} \max _{x \in A \cap S} w_{S}(u(x)) \geq \frac{1}{|\mathscr{C}|} \sum_{S \in \mathscr{C}} \max _{x \in B \cap S} w_{S}(u(x)),
\end{aligned}
$$

with the convention that $\max _{x \in \emptyset} w_{S}(u(x))=0$. Define, then, $\pi \in \triangle(\mathscr{C})$ by $\pi(S):=\frac{1}{|\mathscr{C}|}$ for every $S \in \mathscr{C}$ and $v: X \rightarrow \mathbb{R}$ by $v(x):=w_{S}(u(x))$, where $S \in \mathscr{C}$ is such that $x \in S$, for every $x \in X$. Note that $\langle v, \mathscr{C}, \pi\rangle$ is an additive categorization representation of $\succsim$. 


\section{A.4 Proof of Theorem 5}

Suppose first that $\succsim$ is a relation on $\mathscr{X}$ that has an extreme categorization representation $\langle u, \mathscr{C}, V\rangle$. By Theorem 3 we already know that $\succsim$ is a complete preorder and satisfies Monotonicity and Submodularity and such that $\triangleright$ is asymmetric. We only need to show that satisfies the second part of Strong Compatibility. For that, fix any $x, y \in X$ such that $x \triangleright y$. This can only happen if $\{x, y\} \subseteq S$ for some $S \in \mathscr{C}$. But then we must have $\mathscr{C}=\{X\}$ since $x \neq y$ and $\mathscr{C}$ is an extreme categorization of $X$. In this case, it is clear that for any $z \in X$, neither $\{x, z\} \succ\{x\},\{z\}$ nor $\{y, z\} \succ\{y\},\{z\}$ can happen. We conclude that $\succsim$ satisfies Strong Compatibility.

Conversely, suppose that $\succsim$ is a complete preorder on $\mathscr{X}$ that satisfies Monotonicity, Submodularity and Strong Compatibility. It is clear that Strong Compatibility implies Compatibility. By Theorem 3, we know that $\succsim$ has a categorization representation $\langle u, \mathscr{C}, V\rangle$. Recall that in the proof of Theorem 3 we have defined $\mathscr{C}$ by $\mathscr{C}:=\left\{S_{x}: x \in X\right\}$ where $S_{x}:=\{y \in X: x I y\}$ and $I$ is an equivalence relation on $X$ given by $x I y$ if, and only if, $\{x\} \sim\{x, y\}$ or $\{y\} \sim\{x, y\}$. If $\triangleright=\emptyset$, then it is clear that $S_{x}=\{x\}$ for every $x \in X$. If $\triangleright \neq \emptyset$, we need the following claim:

Claim 1. The relation $I$ is complete.

Proof. Fix any $x, y \in X$. Since $\triangleright \neq \emptyset$, there exist $z, w \in X$ such that $z \triangleright w$. If $x=z$ or $x=w$, then Strong Compatibility implies that $\{x\} \sim\{x, y\}$ or $\{y\} \sim\{x, y\}$, so that $x I y$. Otherwise, Strong Compatibility implies that $\{x\} \sim\{x, z\}$ or $\{z\} \sim\{x, z\}$. In the first case, we get that $x \triangleright z$ and in the second we get that $z \triangleright x$. Now Strong Compatibility implies that $\{x\} \sim\{x, y\}$ or $\{y\} \sim\{x, y\}$ and, again, we learn that $x I y$.

Since $I$ is complete and symmetric, it is clear that $S_{x}=\{X\}$ for every $x \in X$. We conclude that $\langle u, \mathscr{C}, V\rangle$ is an extreme categorization representation of $\succsim$. 


\section{Bibliography}

AGUIAR, V. Random categorization and bounded rationality. mimeo, University of Western Ontario, 2016.

AL-NAJJAR, N. I.; PAI, M. M. Coarse decision making and overfitting. Journal of Economic Theory, Elsevier, v. 150, p. 467-486, 2014.

AZRIELI, Y. Categorizing others in a large game. Games and Economic Behavior, Elsevier, v. 67, n. 2, p. 351-362, 2009.

AZRIELI, Y. Categorization and correlation in a random-matching game. Journal of Mathematical Economics, Elsevier, v. 46, n. 3, p. 303-310, 2010.

BARBERÀ, S.; GRODAL, B. Preference for flexibility and the opportunities of choice. Journal of Mathematical Economics, Elsevier, v. 47, n. 3, p. 272-278, 2011.

BARBERIS, N.; SHLEIFER, A. Style investing. Journal of financial Economics, Elsevier, v. 68, n. 2, p. 161-199, 2003.

BARBOS, A. Context effects: A representation of choices from categories. Journal of Economic Theory, Elsevier, v. 145, n. 3, p. 1224-1243, 2010.

BØLSTAD, J.; DINAS, E. A categorization theory of spatial voting: How the center divides the political space. British Journal of Political Science, Cambridge Univ Press, p. $1-22,2016$.

CHEREPANOV, V.; FEDDERSEN, T.; SANDRONI, A. Rationalization. Theoretical Economics, Wiley Online Library, v. 8, n. 3, p. 775-800, 2013.

COLLINS, N. A. et al. Categorization-based spatial voting. Quarterly Journal of Political Science, now publishers, v. 5, n. 4, p. 357-370, 2011.

FOSTER, J. Notes on effective freedom. mimeo, Vanderbilt University, Vanderbilt University, 1993.

FURTADO, B. A.; NASCIMENTO, L.; RIELLA, G. Rational choice with categories. mimeo, Universidade de Brasilia, 2017.

KOOPMANS, T. C. On flexibility of future preference. [S.l.], 1962.

KREPS, D. M. A representation theorem for "preference for flexibility". Econometrica, JSTOR, v. 47, n. 3, p. 565-577, 1979.

LLERAS, J. S. et al. When more is less: Limited consideration. mimeo, University of Michigan, 2016.

MANZINI, P.; MARIOTTI, M. Categorize then choose: Boundedly rational choice and welfare. Journal of the European Economic Association, Wiley Online Library, v. 10, n. 5, p. 1141-1165, 2012. 
MANZINI, P.; MARIOTTI, M. Stochastic choice and consideration sets. Econometrica, Wiley Online Library, v. 82, n. 3, p. 1153-1176, 2014.

MASATLIOGLU, Y.; NAKAJIMA, D.; OZBAY, E. Y. Revealed attention. The American Economic Review, American Economic Association, v. 102, n. 5, p. 2183-2205, 2012.

MOHLIN, E. Optimal categorization. Journal of Economic Theory, Elsevier, v. 152, p. 356-381, 2014.

NEHRING, K. Preference for flexibility in a savage framework. Econometrica, JSTOR, v. 67, n. 1, p. 101-119, 1999.

PATTANAIK, P. K.; XU, Y. On ranking opportunity sets in terms of freedom of choice. Recherches Économiques de Louvain/Louvain Economic Review, JSTOR, v. 56, n. 3/4, p. 383-390, 1990.

PESKI, M. Prior symmetry, similarity-based reasoning, and endogenous categorization. Journal of Economic Theory, Elsevier, v. 146, n. 1, p. 111-140, 2011.

PUPPE, C. An axiomatic approach to "preference for freedom of choice". Journal of Economic Theory, Elsevier, v. 68, n. 1, p. 174-199, 1996.

SEN, A. K. Welfare, preference and freedom. Journal of Econometrics, Elsevier, v. 50, n. 1, p. 15-29, 1991.

SEN, A. K. Maximization and the act of choice. Econometrica, JSTOR, v. 65, n. 4, p. 745-779, 1997.

WAKKER, P. P. Additive representations of preferences: A new foundation of decision analysis. [S.l.]: Springer Science \& Business Media, 2013. v. 4.

ZHANG, J. Stochastic choice with categorization. mimeo, California Institute of Technology, 2016. 